\title{
Vector meson form factors and their quark-mass dependence
}

\author{
M. S. Bhagwat ${ }^{1}$ and P. Maris ${ }^{2}$ \\ ${ }^{1}$ Physics Division, Argonne National Laboratory, Argonne, IL 60439, U.S.A. \\ ${ }^{2}$ Department of Physics and Astronomy, University of Pittsburgh, Pittsburgh, PA 15260, U.S.A.
}

\begin{abstract}
The electromagnetic form factors of vector mesons are calculated in an explicitly Poincaré covariant formulation, based on the Dyson-Schwinger equations of $\mathrm{QCD}$, that respects electromagnetic current conservation, and unambiguously incorporates effects from vector meson poles in the quark-photon vertex. This method incorporates a 2-parameter effective interaction, where the parameters are constrained by the experimental values of chiral condensate and $f_{\pi}$. This approach has successfully described a large amount of light-quark meson experimental data, e.g. ground state pseudoscalar masses and their electromagnetic form factors; ground state vector meson masses and strong and electroweak decays. Here we apply it to predict the electromagnetic properties of vector mesons. The results for the static properties of the $\rho$-meson are: charge radius $\left\langle r_{\rho}^{2}\right\rangle=0.54 \mathrm{fm}^{2}$, magnetic moment $\mu=2.01$, and quadrupole moment $\mathcal{Q}=-0.41$. We investigate the quark mass dependence of these static properties and find that our results at the charm quark mass are in agreement with recent lattice simulations. The charge radius decreases with increasing quark mass, but the magnetic moment is almost independent of the quark mass.
\end{abstract}

PACS numbers: 11.10.St, 13.40.Gp, 14.40.-n

\section{INTRODUCTION}

Hadron form factors provide an important tool for understanding the structure of bound states in QCD. The coupling of a (virtual) photon to a composite particle depends on its internal structure. Even static properties such as the charge radius and magnetic moment are sensitive to the underlying QCD dynamics. Thus it is not surprising that there have been numerous studies of the electromagnetic form factors of the nucleon, both theoretically and experimentally. Also the form factors of pseudoscalar mesons, in particular of the pion, have been studied extensively [1, 2, 3, 4, 5, 6, 7, 8, 9, 10, 11]. There are fewer studies of the vector meson form factors 12,13 , 14, 15], though in recent years there has been a renewed interest in these form factors [16, 17, 18, 19, 20, 21]. However, the results of these theoretical studies appear to suffer from a rather large model dependence, e.g. the results for the quadrupole moment $G_{\mathrm{Q}}(0)$ differ by a factor of two between different theoretical calculations, and the situation gets worse as one moves away from $Q^{2}=0[19]$.

Here, we calculate the electromagnetic form factors of the $\rho$ and both the neutral and charged $K^{\star}$ mesons using the model proposed in Ref. 22]. The parameters of this model were adjusted to reproduce the experimental values for the chiral condensate, the pion mass and decay constant, and the kaon mass; calculations of other mesonic observables are predictions. The obtained vector meson masses and decay constants are in agreement with data 22] as are the results for the strong decays of the vector mesons 23]. The pion and kaon electromagnetic form factors 6, 7] are also in excellent agreement with experiments [10, 11], as are the transition form factors such as the $\rho \rightarrow \pi \gamma$ [24]. This proclivity of theoretical results to experimental data provides good reason to expect that the model will accurately describe the electromagnetic form factors of light vector mesons.
In Sec. II we review the Dyson-Schwinger equations used to calculate the quark propagators and the meson Bethe-Salpeter amplitudes. Next, we discuss the truncation and the model for the effective interaction, and the results for the meson masses, decay constants, and their quark mass dependence. We explicitly demonstrate frame independence: our results for physical observables are independent of the total meson momentum. This is important since in form factor calculations at least one of the mesons is moving. In Sec. IV] we briefly discuss the general form of vector meson form factors and the method of calculation; an essential element of our calculation is the treatment of the quark-photon vertex. We present our numerical results for the form factors of the $\rho$ and of both the neutral and charged $K^{\star}$ mesons in Sec. V] as well as the mass dependence of the static electromagnetic properties of equal-mass vector mesons; we also compare our results with other calculations and with recent lattice simulations. Finally, some of the details of our calculation are given in the appendix.

\section{DYSON-SCHWINGER EQUATIONS OF QCD}

The Dyson-Schwinger equations [DSEs] are the equations of motion of a quantum field theory. They form an infinite hierarchy of coupled integral equations for the Green functions ( $n$-point functions) of the theory. Bound states (mesons, baryons) appear as poles in the Green functions. Thus, a study of the poles in $n$-point functions using the set of DSEs will tell us about properties of hadrons. For recent reviews on the DSEs and their use in hadron physics, see Refs. [25, 26, 27, 28]. 


\section{A. Quark propagator}

The exact DSE for the quark propagator is ${ }^{1}$

$$
\begin{aligned}
S(p)^{-1}= & i \not p Z_{2}+m_{q}(\zeta) Z_{4}+ \\
& Z_{1} \int_{k} g^{2} D_{\mu \nu}(q) \gamma_{\mu} \frac{\lambda^{i}}{2} S(k) \Gamma_{\nu}^{i}(k, p),
\end{aligned}
$$

where $D_{\mu \nu}(q=k-p)$ is the renormalized dressed gluon propagator, and $\Gamma_{\nu}^{i}(k, p)$ is the renormalized dressed quark-gluon vertex. The notation $\int_{k}$ stands for $\int^{\Lambda} d^{4} k /(2 \pi)^{4}$. For divergent integrals a translationally invariant regularization is necessary; the regularization scale $\Lambda$ is to be removed at the end of all calculations, after renormalization, and will be suppressed henceforth.

The solution of Eq. (1) can be written as

$$
S(p)=\frac{Z\left(p^{2}\right)}{i \not p+M\left(p^{2}\right)},
$$

renormalized according to $S(p)^{-1}=i \not p+m(\zeta)$ at a sufficiently large spacelike $\zeta^{2}$, with $m(\zeta)$ the current quark mass at the scale $\zeta$. Both the propagator, $S(p)$, and the vertex, $\Gamma_{\mu}^{i}$, depend on the quark flavor, although we have not indicated this explicitly. The renormalization constants $Z_{2}$ and $Z_{4}$ depend on the renormalization point and on the regularization mass-scale, but not on flavor: in our analysis we employ a flavor-independent renormalization scheme.

\section{B. Mesons}

Bound states correspond to poles in $n$-point functions: for example a meson appears as a pole in the 2-quark, 2-antiquark Green function $G^{(4)}=\left\langle 0\left|q_{1} q_{2} \overline{q_{1}} \overline{q_{2}}\right| 0\right\rangle$. In the vicinity of a meson, i.e. in the neighborhood of $P^{2}=-M^{2}$ with $M$ being the meson mass, such a Green function behaves like

$$
G^{(4)} \sim \frac{\chi\left(p_{\mathrm{out}}, p_{\mathrm{in}} ; P\right) \bar{\chi}\left(k_{\mathrm{in}}, k_{\mathrm{out}} ; P\right)}{P^{2}+M^{2}},
$$

where $P$ is the total 4-momentum of the meson, $p_{\text {out }}$ and $p_{\text {in }}$ are the 4 -momenta of the outgoing quark and incoming quark respectively, and similarly for $k_{\text {in }}$ and $k_{\text {out }}$. Momentum conservation relates these momenta: $p_{\text {out }}-p_{\text {in }}=P=k_{\text {out }}-k_{\text {in }}$.

The function $\chi\left(p_{\text {out }}, p_{\text {in }} ; P\right)$ describes the coupling of the bound state to a dressed quark and antiquark. It satisfies the homogeneous Bethe-Salpeter equation [BSE]

$$
\begin{aligned}
& \Gamma\left(p_{\text {out }}, p_{\text {in }} ; P\right)= \\
& \quad \int_{k} K\left(p_{\text {out }}, p_{\text {in }} ; k_{\text {out }}, k_{\text {in }}\right) \chi\left(k_{\text {out }}, k_{\text {in }} ; P\right),
\end{aligned}
$$

\footnotetext{
${ }^{1}$ We use Euclidean metric $\left\{\gamma_{\mu}, \gamma_{\nu}\right\}=2 \delta_{\mu \nu}, \gamma_{\mu}^{\dagger}=\gamma_{\mu}$ and $a \cdot b=$ $\sum_{i=1}^{4} a_{i} b_{i}$.
}

at discrete values $P^{2}=-M^{2}$ of the total meson 4momentum $P$. Here $\Gamma$ is the Bethe-Salpeter amplitude [BSA]

$$
\Gamma\left(k_{\text {out }}, k_{\text {in }} ; P\right)=S\left(k_{\text {out }}\right)^{-1} \chi\left(k_{\text {out }}, k_{\text {in }} ; P\right) S\left(k_{\text {in }}\right)^{-1} \text {, }
$$

and the kernel $K$ is the $q \bar{q}$ irreducible quark-antiquark scattering kernel.

The meson BSA is normalized according to

$$
\begin{aligned}
& 2 P_{\mu}=N_{c} \frac{\partial}{\partial Q_{\mu}}\{ \\
& \int_{k, q} \operatorname{Tr}\left[\bar{\chi}\left(k_{\text {in }}, k_{\text {out }}\right) K\left(\tilde{k}_{\text {out }}, \tilde{k}_{\text {in }} ; \tilde{q}_{\text {out }}, \tilde{q}_{\text {in }}\right) \chi\left(q_{\text {out }}, q_{\text {in }}\right)\right] \\
& \left.\quad+\int_{k} \operatorname{Tr}\left[\bar{\Gamma}\left(k_{\text {in }}, k_{\text {out }}\right) S\left(\tilde{k}_{\text {out }}\right) \Gamma\left(k_{\text {out }}, k_{\text {in }}\right) S\left(\tilde{k}_{\text {in }}\right)\right]\right\}\left.\right|_{Q=P}
\end{aligned}
$$

at $P^{2}=-M^{2}$, with $\tilde{k}_{\text {out }}-\tilde{k}_{\text {in }}=Q=\tilde{q}_{\text {out }}-\tilde{q}_{\text {in }}$. The properly normalized BSA $\Gamma\left(p_{\text {out }}, p_{\text {in }} ; P\right)$ [or equivalently, $\left.\chi\left(p_{\text {out }}, p_{\text {in }} ; P\right)\right]$ completely describes the meson as a $q \bar{q}$ bound state. Mesons of different spins and parity are characterized by different Dirac structures, e.g. the BSA of massive vector mesons can be decomposed into eight Dirac structures [22]

$$
\begin{gathered}
\Gamma_{\mu}^{\mathrm{V}}(k+\eta P, k-(1-\eta) P ; P)= \\
\sum_{i=1}^{8} f^{i}\left(k^{2}, k \cdot P ; \eta\right) T_{\mu}^{i}(k, P),
\end{gathered}
$$

where $T_{\mu}^{i}(k, P)$ are eight independent transverse Dirac tensors. The invariant amplitudes $f^{i}$ are Lorentz scalar functions of $k^{2}$ and $k \cdot P$, and depend on the momentum partitioning parameter $\eta$. Physical observables however are independent of $\eta$.

\section{Rainbow-ladder truncation}

A viable truncation of the infinite set of DSEs has to respect relevant (global) symmetries of QCD such as chiral symmetry, Poincaré covariance, and renormalization group invariance. For electromagnetic interactions the truncation should also respect current conservation. These properties are built into the rainbow-ladder truncation [6, 7, 29, 30, 31]. In this scheme, the kernel $K$ of the meson BSE is replaced by an (effective) one-gluon exchange

$$
\begin{aligned}
& K\left(p_{\mathrm{out}}, p_{\mathrm{in}} ; k_{\mathrm{out}}, k_{\mathrm{in}}\right) \\
& \rightarrow-4 \pi \alpha\left(q^{2}\right) D_{\mu \nu}^{\text {free }}(q) \frac{\lambda^{i}}{2} \gamma_{\mu} \otimes \frac{\lambda^{i}}{2} \gamma_{\nu},
\end{aligned}
$$

where $q=p_{\text {out }}-k_{\text {out }}=p_{\text {in }}-k_{\text {in }}$, and $\alpha\left(q^{2}\right)$ is an effective running coupling; $D_{\mu \nu}^{\text {free }}(q)$ is the free gluon propagator; and we choose to work in Landau gauge. The corresponding rainbow truncation of the quark DSE is

$$
Z_{1} g^{2} D_{\mu \nu}(q) \Gamma_{\nu}^{i}(k, p) \rightarrow 4 \pi \alpha\left(q^{2}\right) D_{\mu \nu}^{\text {free }}(q) \gamma_{\nu} \frac{\lambda^{i}}{2} .
$$


This truncation is the first term in a systematic expansion [29] of the quark-antiquark scattering kernel $K$; asymptotically, it reduces to leading-order perturbation theory. Furthermore, these two truncations are mutually consistent in the sense that the combination produces vector and axial-vector vertices satisfying their respective Ward identities. In the axial case, this ensures that in the chiral limit the ground state pseudoscalar mesons are the massless Goldstone bosons associated with chiral symmetry breaking [30, 31]. In the vector case, this ensures, in combination with impulse approximation, electromagnetic current conservation [6, 7].

\section{MODEL CALCULATIONS}

The ultraviolet behavior of the effective running coupling is dictated by the one-loop renormalization group equation; the infrared behavior of the effective interaction is modeled, and constrained by phenomenology. Here, we employ the model of Ref. [22] for $\alpha\left(q^{2}\right)$

$$
\begin{aligned}
\frac{4 \pi \alpha\left(q^{2}\right)}{k^{2}}= & \frac{4 \pi^{2} D k^{2}}{\omega^{6}} \mathrm{e}^{-k^{2} / \omega^{2}} \\
& +\frac{4 \pi^{2} \gamma_{m} \mathcal{F}\left(k^{2}\right)}{\frac{1}{2} \ln \left[\tau+\left(1+k^{2} / \Lambda_{\mathrm{QCD}}^{2}\right)^{2}\right]},
\end{aligned}
$$

with $\mathcal{F}(s)=\left(1-\exp \frac{-s}{4 m_{t}^{2}}\right) / s, \gamma_{m}=12 /\left(33-2 N_{f}\right)$, and fixed parameters $m_{t}=0.5 \mathrm{GeV}, \tau=\mathrm{e}^{2}-1, N_{f}=4$, and $\Lambda_{\mathrm{QCD}}=0.234 \mathrm{GeV}$. The remaining parameters $\omega$ and $D$ were fitted in Ref. 22] to reproduce $f_{\pi}$ and the chiral condensate: $\omega=0.4 \mathrm{GeV}$ and $D=0.93 \mathrm{GeV}^{2}$.

\section{A. Results for light quarks}

With this model, we obtain good agreement with the experimental values for the light pseudoscalar and vector meson masses and leptonic decay constants, see Table I. The current quark masses $m_{u / d}=3.7 \mathrm{MeV}$ and $m_{s}=83.8 \mathrm{MeV}$ at the renormalization point $\zeta=19 \mathrm{GeV}$ were fitted [22] to the pion and kaon mass respectively. Using the one-loop expression to evolve these masses down to $\zeta=2 \mathrm{GeV}$ gives $m_{u / d}(1 \mathrm{GeV})=5.0 \mathrm{MeV}$ and $m_{s}(2 \mathrm{GeV})=118 \mathrm{MeV}$.

These results show little sensitivity to variations in the model parameters [36], as long as the integrated strength of the effective interaction is strong enough to generate an acceptable amount of chiral symmetry breaking, as indicated by the chiral condensate. This is not true for heavier states consisting of light quarks: e.g. the radially excited pion is quite sensitive to details of the interaction [37].

Not only the meson masses and leptonic decay constants, but also a wide range of other observables agree with experiments, without adjusting any of the parameters, see [27] and references therein. In particular

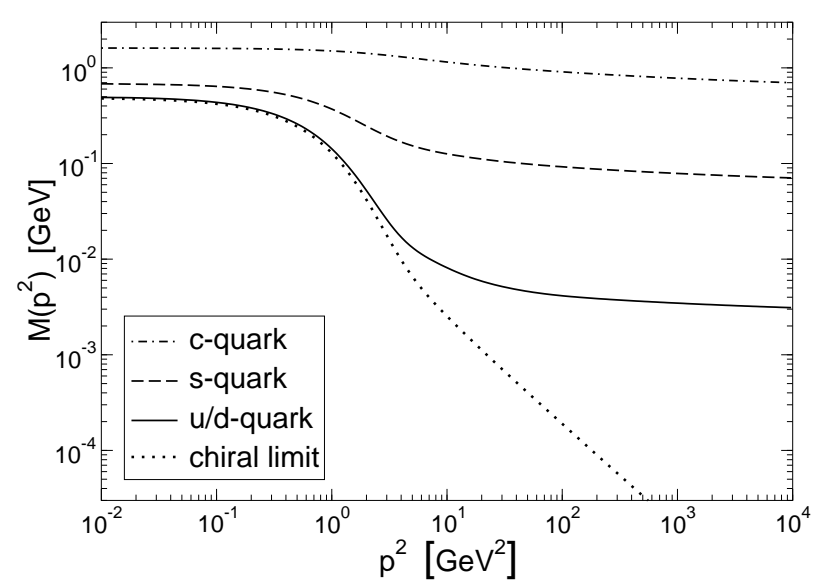

FIG. 1: Dynamical quark mass function using the rainbowladder truncation of Ref. 22].

$F_{\pi}\left(Q^{2}\right)$ [6, 7, 10, 11] and the $\rho-\pi-\gamma, \omega-\pi-\gamma$, and $\pi-\gamma \gamma$ form factors 24] are well described by this model. We therefore expect the model to describe the electromagnetic form factors of light vector mesons quite accurately as well.

The corresponding quark propagator functions are shown in Fig. 1. These predictions for the quark mass function have been semi-quantitatively confirmed in recent lattice simulations of QCD [38, 39, 40]. Point-wise agreement for a range of quark masses requires this interaction to be flavor-dependent [41], and dressing the

TABLE I: DSE results [22, 32] for the pseudoscalar and vector meson masses and decay constants, together with experimental data from Ref. [33], unless indicated otherwise. All entries are in $\mathrm{GeV}$.

\begin{tabular}{lll}
\hline & $\begin{array}{l}\text { experiment } \\
\text { (estimates) }\end{array}$ & $\begin{array}{l}\text { calculated } \\
\text { ( }{ }^{\dagger} \text { fitted) }\end{array}$ \\
\hline$m_{u / d}(\zeta=2 \mathrm{GeV})$ & 0.003 to 0.006 & 0.005 \\
$m_{s}(\zeta=2 \mathrm{GeV})$ & $0.095(25)$ & 0.118 \\
\hline$m_{c}\left(\zeta=m_{c}\right)$ & $1.25(9)$ & 1.30 \\
\hline$M_{\pi}$ & $0.135,0.140$ & $0.138^{\dagger}$ \\
$f_{\pi}$ & 0.131 & $0.131^{\dagger}$ \\
$M_{K}$ & 0.496 & $0.497^{\dagger}$ \\
$f_{K}$ & 0.160 & 0.155 \\
\hline$M_{\rho}, M_{\omega}$ & $0.776,0.783$ & 0.742 \\
$f_{\rho}, f_{\omega}$ & $0.221(2), 0.195(4)$ & 0.207 \\
$M_{K^{\star}}$ & 0.892 & 0.936 \\
$f_{K^{\star}}$ & $0.224(11)$ & 0.241 \\
$M_{\phi}$ & 1.020 & 1.074 \\
$f_{\phi}$ & $0.229(4)$ & 0.259 \\
\hline$M_{\eta_{c}}$ & 2.980 & 2.91 \\
$f_{\eta_{c}}$ & $0.335(75)[35]$ & 0.38 \\
$M_{J / \Psi}$ & 3.097 & $3.10^{\dagger}$ \\
$f_{J / \Psi}$ & $0.416(6)$ & 0.42 \\
\hline
\end{tabular}




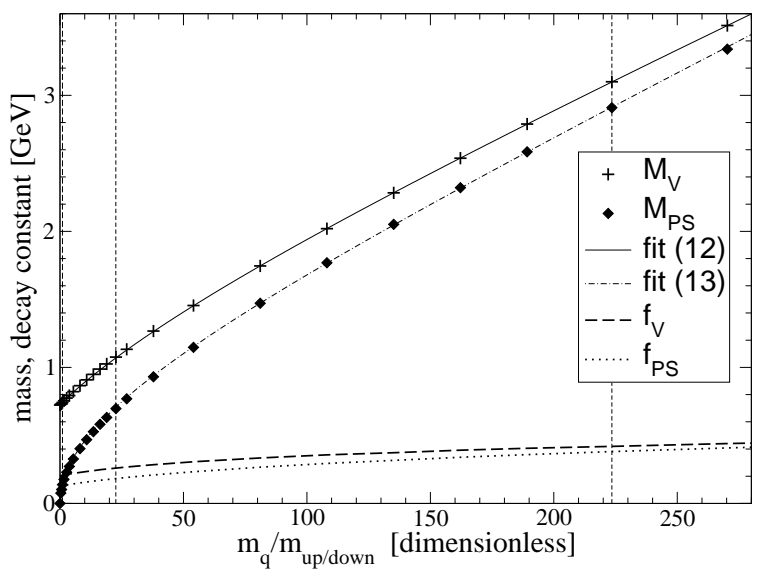

FIG. 2: Pseudoscalar and vector meson masses and leptonic decay constants as function of the current quark mass (normalized to the physical up/down quark mass). Vertical dashed lines indicate the up/down, strange, and charm quark masses.

quark-gluon vertex $\Gamma_{\nu}^{i}(q, p)$ ensures this dependence. The consequences of a dressed vertex for the meson BSEs are also currently being explored and indications are that in the pseudoscalar and vector channels, the effects are small [29, 42, 43]. The non-trivial infrared structure of the quark-gluon vertex is under investigation using both lattice simulations [44, 45, 46] and non-perturbative DSE methods [47, 48], but these studies are not yet conclusive.

\section{B. Quark-mass dependence}

In recent years, this model has been extended [32, 49] to the charm and bottom quarks as well, in an attempt to describe both the light mesons and heavy quarkonia within one framework. In Fig. 2 we show our results for the vector meson mass and decay constant as function of the current quark mass; for comparison we also include the evolution of the corresponding pseudoscalar meson mass and decay constant. In this and subsequent figures, we normalize the current quark masses by the physical up and down quark masses of our model; for the strange quark we have $m_{s} \approx 23 m_{u / d}$.

The charm quark mass $m_{c}=0.827 \mathrm{GeV}$ at the renormalization point $\zeta=19 \mathrm{GeV}$ is fixed by the experimental value of $J / \Psi$ mass. Again, using one-loop evolution this corresponds to $m_{c}=1.30 \mathrm{GeV}$ at $\zeta=m_{c}$, see Table @ The decay constant for the $J / \Psi$ is in good agreement with data, and the resulting post-dictions for the $\eta_{c}$ mass and decay constant are in reasonable agreement with the data as well.

On a limited domain, both the vector and the pseudoscalar meson masses can be fitted reasonably well by

$$
M_{\text {meson }}^{2}=C_{0}+C_{1} m_{q}+C_{2} m_{q}^{2},
$$

where $m_{q}$ is the current quark mass at our renormal- ization point $\zeta=19 \mathrm{GeV}$. For the mass region we are interested in here, from the chiral limit up to the charm quark mass, the fit parameters are

$$
\begin{aligned}
M_{\mathrm{PS}}: & C_{0}=0, \quad C_{1}=5.49, \quad C_{2}=5.77, \\
M_{\mathrm{V}}: & C_{0}=0.53, \quad C_{1}=6.93, \quad C_{2}=4.88,
\end{aligned}
$$

as illustrated in Fig. 2, For larger quark masses, the fit parameters for the pseudoscalar and vector mesons become closer and closer to each other: a fit on the domain $m_{c}<m_{q}<2 m_{b}$ gives $C_{0}=-1.3$ and $C_{1}=7.92$ for the pseudoscalar mesons, and $C_{0}=-0.2$ and $C_{1}=8.02$ for the vector mesons, with a common parameter $C_{2}=4.46$. This reflects the fact that in the heavy-quark limit, the pseudoscalar and vector mesons become degenerate: in the limit $m_{q} \rightarrow \infty$ the above fit gives $M_{\mathrm{V}}-M_{\mathrm{PS}} \rightarrow$ $\frac{1}{2}\left(C_{1}^{\mathrm{V}}-C_{1}^{\mathrm{PS}}\right) / \sqrt{C_{2}} \approx 0$.

The leptonic decay constants increase with the current quark mass, both for the pseudoscalar and for the vector mesons. Based on the experimental partial decay width of vector mesons, it was conjectured [50] that the vector meson decay constants increase with quark mass as $f_{\mathrm{V}} \propto \sqrt{M_{\mathrm{V}}}$. On the other hand [51], Coulomb-potential models typically give $f_{\mathrm{V}} \propto M_{\mathrm{V}}$, whereas a linear (confining) potential produces $f_{\mathrm{V}} \sim$ constant. Our numerical results suggest that both $f_{\mathrm{V}}$ and $f_{\mathrm{PS}}$ increase approximately linearly with quark mass, at least for masses in the $m_{c}$ to twice $m_{b}$ range [52]. This is Coulomb-potentiallike behavior, which may be natural since the effective interaction, Eq.10, reduces to one-gluon exchange in the ultraviolet region. However, further investigations are needed in order to determine the true asymptotic behavior of the decay constants.

\section{Frame independence}

The BSE is usually solved in the rest-frame of the meson. However, the calculation of electromagnetic form factors in any reference frame entails either the initial meson, or the final meson, or both, to have non-zero 3momentum. In a method that is not Poincaré covariant the wave functions for the moving meson would have to be boosted. One of the advantages of the DSE approach to hadron physics is its manifest Poincaré covariance.

As an explicit demonstration, we calculate the static $\pi$ and $\rho$ properties in a moving frame ${ }^{2} P_{\mu}=(q, 0,0, i E)$ where $q$ is the 3 -momentum of the moving meson [49]. Within this frame we solve again the homogeneous BSE, Eq. (4), and calculate the corresponding electroweak decay constant. Numerically this is a demanding task, since the Lorentz scalar functions of Eq. (77) are now functions of a radial variable $k^{2}$ and two angles

$$
k \cdot P=i k E \cos \alpha+k q \sin \alpha \cos \beta,
$$

\footnotetext{
${ }^{2}$ In the Euclidean metric that we are using here, the rest-frame is characterized by $P_{\mu}=(0,0,0, i M)$.
} 


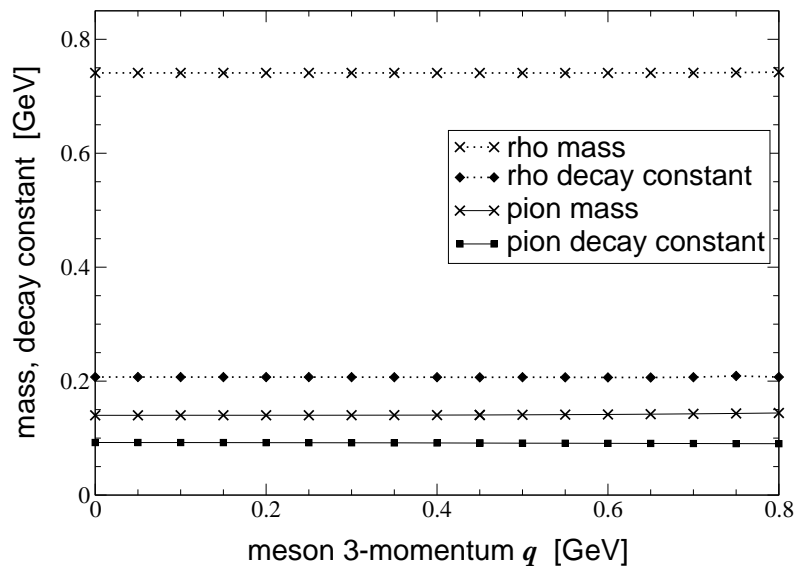

FIG. 3: Pion and $\rho$ mass and decay constant calculated in a moving frame, as function of the meson 3-momentum (Figure adapted from Ref. [49]).

and the integral equation has to be solved in the three independent variables $k^{2}, \alpha$, and $\beta$. With current computer resources, this can be done without further approximations, and the results, shown in Fig. 3. are indeed independent of the meson 3-momentum, illustrating that this approach is indeed Poincaré covariant. We can now use this same approach to calculate meson form factors in an explicitly covariant manner.

\section{ELECTROMAGNETIC FORM FACTORS}

\section{A. Vector meson form factors}

Consider the electromagnetic current of a vector meson with incoming meson momentum $P_{\rho}^{-}=P_{\rho}-\frac{1}{2} Q_{\rho}$, outgoing meson momentum $P_{\sigma}^{+}=P_{\sigma}+\frac{1}{2} Q_{\sigma}$, and incoming photon momentum $Q_{\mu}$. With this notation, the general form for the coupling of a photon to a vector meson can be written as [15, 53 ]

$$
\begin{aligned}
\Lambda_{\mu, \rho \sigma}(P, Q)= & -\sum_{j=1}^{3} T_{\mu \rho \sigma}^{[j]}(P, Q) F_{j}\left(Q^{2}\right) \\
T_{\mu, \rho \sigma}^{[1]}(P, Q)= & 2 P_{\mu} \mathcal{P}_{\rho \gamma}^{T}\left(P^{-}\right) \mathcal{P}_{\gamma \sigma}^{T}\left(P^{+}\right) \\
T_{\mu, \rho \sigma}^{[2]}(P, Q)= & \left(Q_{\rho}-P_{\rho}^{-} \frac{Q^{2}}{2 M^{2}}\right) \mathcal{P}_{\mu \sigma}^{T}\left(P^{+}\right) \\
& -\left(Q_{\sigma}+P_{\sigma}^{+} \frac{Q^{2}}{2 M^{2}}\right) \mathcal{P}_{\mu \rho}^{T}\left(P^{-}\right), \\
T_{\mu, \rho \sigma}^{[3]}(P, Q)= & \frac{P_{\mu}}{M^{2}}\left(Q_{\rho}-P_{\rho}^{-} \frac{Q^{2}}{2 M^{2}}\right) \\
& \times\left(Q_{\sigma}+P_{\sigma}^{+} \frac{Q^{2}}{2 M^{2}}\right)
\end{aligned}
$$

where

$$
\mathcal{P}_{\mu \nu}^{T}(k)=\delta_{\mu \nu}-\frac{k_{\mu} k_{\nu}}{k^{2}}
$$

is the transverse projector. The vector meson is on-shell: $\left(P^{-}\right)^{2}=\left(P^{+}\right)^{2}=-M^{2}$, where $M$ is the mass of the vector meson, and thus $P^{2}+\frac{1}{4} Q^{2}=-M^{2}$ and $P \cdot Q=0$. This coupling obeys the following relations

$$
\begin{aligned}
& P_{\rho}^{+} \Lambda_{\mu \rho \sigma}\left(p, p^{\prime}\right)=0, \\
& P_{\sigma}^{-} \Lambda_{\mu \rho \sigma}\left(p, p^{\prime}\right)=0, \\
& Q_{\mu} \Lambda_{\mu \rho \sigma}\left(p, p^{\prime}\right)=0 .
\end{aligned}
$$

The first two equations simply reflect that the (massive) vector mesons are transverse; the last equation follows from current conservation.

The electric, magnetic, and quadrupole form factors $G_{\mathrm{E}}, G_{\mathrm{M}}, G_{\mathrm{Q}}$ can be expressed in terms of these scalar functions $F_{i}$

$$
\begin{aligned}
G_{\mathrm{E}}\left(Q^{2}\right) & =F_{1}\left(Q^{2}\right)+\frac{2}{3} \frac{Q^{2}}{4 M^{2}} G_{\mathrm{Q}}\left(Q^{2}\right) \\
G_{\mathrm{M}}\left(Q^{2}\right) & =-F_{2}\left(Q^{2}\right) \\
G_{\mathrm{Q}}\left(Q^{2}\right) & =F_{1}\left(Q^{2}\right)+F_{2}\left(Q^{2}\right)+\left(1+\frac{Q^{2}}{4 M^{2}}\right) F_{3}\left(Q^{2}\right) .
\end{aligned}
$$

The electric monopole moment (i.e. the electric charge), magnetic dipole moment and the electric quadrupole moment follow from the values of these form factors in the limit $Q^{2} \rightarrow 0$

$$
\begin{aligned}
G_{\mathrm{E}}\left(Q^{2}=0\right) & =1, \\
G_{\mathrm{M}}\left(Q^{2}=0\right) & =\mu, \\
G_{\mathrm{Q}}\left(Q^{2}=0\right) & =\mathcal{Q} .
\end{aligned}
$$

Here the magnetic moment $\mu$ and the quadrupole moment $\mathcal{Q}$ are introduced; the electric charge is 1 in terms of the fundamental charge unit $e$. For point-like vector particles, the magnetic and quadrupole moments are $\mu=2$ in units of $e / 2 M_{\mathrm{V}}$ and $\mathcal{Q}=-1$ in units of $e / M_{\mathrm{V}}^{2}$, respectively [54].

\section{B. Impulse approximation}

The generalized impulse approximation allows electromagnetic processes to be described in terms of dressed quark propagators, bound state BSAs, and the dressed $q \bar{q} \gamma$-vertex, see Fig. 4. In combination with ladderrainbow truncation for the vertices and the quark propagators, it ensures electromagnetic current conservation [6, 7], see also Sec. IVC below. Phenomenologically, this approximation has proved to be very successful in describing the pion electromagnetic form factor [10, 11].

Consider for example the 3-point function describing the coupling of a photon with momentum $Q$ to a vector meson $a \bar{b}$, with initial and final momenta $P \pm Q / 2$. This interaction can be written as the sum of two terms

$$
\Lambda_{\mu, \rho \sigma}^{a \bar{b}}(P, Q)=\hat{Q}^{a} \Lambda_{\mu, \rho \sigma}^{a a \bar{b}}+\hat{Q}^{\bar{b}} \Lambda_{\mu, \rho \sigma}^{a \bar{b} \bar{b}},
$$




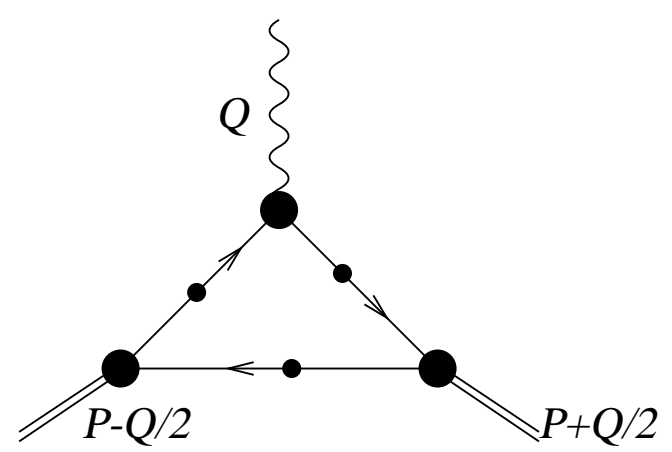

FIG. 4: Meson form factor in impulse approximation

where $\hat{Q}$ is the quark or antiquark electric charge, and where $\Lambda^{a \bar{b} a}(P, Q)$ and $\Lambda^{a \bar{b} \bar{b}}(P, Q)$ describe the coupling of a photon to the quark $(a)$ and antiquark $(\bar{b})$ respectively. In impulse approximation, these couplings are given by

$$
\begin{aligned}
\Lambda_{\mu, \rho \sigma}^{a a \bar{b}}(P, Q)= & i N_{c} \int_{k} \operatorname{Tr}\left[\Gamma_{\mu}^{a}\left(q_{-}, q_{+}\right) \chi_{\rho}^{a \bar{b}}\left(q_{+}, q\right)\right. \\
& \left.\times S^{b}(q)^{-1} \bar{\chi}_{\sigma}^{\bar{b} a}\left(q, q_{-}\right)\right],
\end{aligned}
$$

with $q=k-P / 2$ and $q_{ \pm}=k+P / 2 \pm Q / 2$, and similarly for $\Lambda_{\mu, \rho \sigma}^{a \bar{b} \bar{b}}$.

For the $\rho$ mesons it is sufficient to calculate the coupling of the photon to a single quark, a direct consequence of isospin invariance, while for the $K^{\star}$ mesons we add contributions from photon coupling to the quark and the antiquark. Thus for the charged $K^{\star}$ the form factors are given by

$$
\begin{aligned}
F_{i}^{K^{\star,+}}\left(Q^{2}\right) & =\frac{2}{3} F_{i}^{u u \bar{s}}\left(Q^{2}\right)-\frac{1}{3} F_{i}^{u \bar{s} \bar{s}}\left(Q^{2}\right) \\
& =\frac{2}{3} F_{i}^{u u \bar{s}}\left(Q^{2}\right)+\frac{1}{3} F_{i}^{s s \bar{u}}\left(Q^{2}\right),
\end{aligned}
$$

and similarly for the neutral $K^{\star}$ we have

$$
F_{i}^{K^{\star, 0}}\left(Q^{2}\right)=-\frac{1}{3} F_{i}^{u u \bar{s}}\left(Q^{2}\right)+\frac{1}{3} F_{i}^{s s \bar{u}}\left(Q^{2}\right) .
$$

\section{Quark-photon vertex}

The impulse approximation and rainbow-ladder truncation together lead to current conservation only if the quark-photon vertex $\Gamma_{\mu}$ satisfies the vector WardTakahashi identity [3]

$$
i Q_{\mu} \Gamma_{\mu}\left(k_{+}, k_{-} ; Q\right)=S^{-1}\left(k+\frac{1}{2} Q\right)-S^{-1}\left(k-\frac{1}{2} Q\right) .
$$

This identity can be satisfied by the Ball-Chiu Ansatz [55] for the quark-photon vertex; a more consistent approach, which we follow, is to use the solution of the inhomogeneous BSE for the $q \bar{q} \gamma$-vertex in the ladder truncation [6, 7]

$$
\begin{aligned}
& \Gamma_{\mu}\left(p_{\text {out }}, p_{\text {in }} ; Q\right)=Z_{2} \gamma_{\mu}+\int_{k} K\left(p_{\text {out }}, p_{\text {in }} ; k_{\text {out }}, k_{\text {in }}\right) \\
& \quad \times S\left(k_{\text {out }}\right) \Gamma_{\mu}\left(k_{\text {out }}, k_{\text {in }} ; Q\right) S\left(k_{\text {in }}\right),
\end{aligned}
$$

with $p_{\text {out }}$ and $p_{\text {in }}$ the outgoing and incoming quark momenta, respectively, and similarly for $k_{\text {out }}$ and $k_{\text {in }}$, with $p_{\text {out }}-p_{\text {in }}=k_{\text {out }}-k_{\text {in }}=Q$. The kernel $K$ is the same kernel as used in the meson BSE, defined under Eq. (44).

Note that solutions of the homogeneous version of Eq. (35) define $q \bar{q}$ vector meson bound states with masses $M_{\mathrm{V}}^{2}=-Q^{2}$ at discrete timelike momenta $Q^{2}$. It follows that $\Gamma_{\mu}$ has poles at those locations and, in the neighborhood of $Q^{2}=-M_{\mathrm{V}}^{2}$, behaves like [ $[6]$

$$
\Gamma_{\mu}\left(p_{\text {out }}, p_{\text {in }}\right) \sim \frac{\Gamma_{\mu}^{\mathrm{V}}\left(p_{\text {out }}, p_{\text {in }}\right) f_{\mathrm{V}} M_{\mathrm{V}}}{Q^{2}+M_{\mathrm{V}}^{2}},
$$

where $\Gamma_{\mu}^{\mathrm{V}}$ is the $q \bar{q}$ vector meson BSA, and $f_{\mathrm{V}}$ the corresponding electroweak decay constant. The fact that the dressed $q \bar{q} \gamma$-vertex exhibits these vector meson poles explains the success of naive vector-meson-dominance [VMD] models; the effects of intermediate vector meson states on electromagnetic processes can be unambiguously incorporated by using the properly dressed $q \bar{q} \gamma$ vertex rather than the bare vertex $\gamma_{\mu}[6]$.

\section{DISCUSSION OF NUMERICAL RESULTS}

Numerical solutions for the quark propagator, vector meson BSAs and dressed quark-gluon vertex can now be used in Eq. (30) to calculate the electromagnetic form factors. We explicitly solve the respective (in)homogeneous BSEs for the meson BSAs and for the $q \bar{q} \gamma$-vertex in the corresponding momentum frame, thus avoiding any need for interpolation or extrapolation of the numerical solutions of the BSEs. This does mean that we have to solve the meson BSE for each value of $Q^{2}$, but the advantages of not having to extrapolate the numerical BSAs outweighs the additional numerical effort of repeatedly solving the meson BSE. Further details are given in the appendix.

\section{A. Rho and $K^{\star}$ form factors}

Results for the charge radius,

$$
\left\langle r^{2}\right\rangle=-\left.6 \frac{\partial G_{\mathrm{E}}\left(Q^{2}\right)}{\partial Q^{2}}\right|_{Q^{2}=0},
$$

the magnetic moment $\mu=G_{\mathrm{M}}(0)$, and the magnetic quadrupole moment $\mathcal{Q}=G_{\mathrm{Q}}(0)$, are presented in Table II. Experimental data on these static properties is absent, and so results from other theoretical models are included in the table. 
TABLE II: Results for the $\rho$ and $K^{\star}$ meson charge radii $\left\langle r_{\mathrm{V}}^{2}\right\rangle$ in $\mathrm{fm}^{2}$, magnetic moments, and quadrupole moments, compared to other calculations. For comparison, we also include the results for the pseudoscalar charge radii, calculated within the same model, where available, as well as the experimental pseudoscalar charge radii.

\begin{tabular}{l|c|ccc}
$\pi, \rho$ meson & $r_{\pi}^{2}$ & $r_{\rho}^{2}$ & $\mu$ & $\mathcal{Q}$ \\
\hline current DSE [6, 32] & 0.44 & 0.54 & 2.01 & -0.41 \\
previous DSE [4, 15] & 0.31 & 0.37 & 2.69 & -0.84 \\
Covariant QM [13] & & 0.37 & 2.14 & -0.79 \\
Lightcone QM [5, 17] & 0.43 & 0.27 & 1.92 & -0.43 \\
experimental [33] & $0.452(11)$ & & & \\
\hline$u \bar{s}$ meson & $r_{K}^{2}$ & $r_{K^{\star}}^{2}$ & $\mu$ & $\mathcal{Q}$ \\
\hline current DSE calc. & 0.38 & 0.43 & 2.23 & -0.38 \\
previous DSE [15] & 0.28 & 0.29 & 2.37 & -0.62 \\
experimental [33] & $0.314(35)$ & & & \\
\hline$d \bar{s}$ meson & $r_{K}^{2}$ & $r_{K^{\star}}^{2}$ & $\mu$ & $\mathcal{Q}$ \\
\hline current DSE calc. & -0.09 & -0.08 & -0.26 & 0.01 \\
previous DSE [15] & -0.03 & -0.05 & -0.40 & 0.11 \\
experimental [33] & $-0.077(10)$ & \multicolumn{4}{|c}{} \\
\hline$c \bar{c}$ meson & $r_{\eta_{c}}^{2}$ & $r_{J / \psi}^{2}$ & $\mu$ & $\mathcal{Q}$ \\
\hline current DSE [32] & $0.048(4)$ & $0.052(3)$ & $2.13(4)$ & $-0.28(1)$ \\
lattice [20] & $0.063(1)$ & $0.066(2)$ & $2.10(3)$ & $-0.23(2)$
\end{tabular}

Our calculations give a significantly larger value for the charge radius of the $\rho$ and the charged $K^{\star}$ mesons compared to a previous DSE calculation [15], see Table III. This is the case not only for the vector mesons, but also for the pion and kaon charge radii. This difference can largely be attributed [6] to the fact that we employ a dressed quark-photon vertex that has poles in the neighborhood of $Q^{2}=-M_{\mathrm{V}}^{2}$. Thus the effects from intermediate vector meson states are unambiguously included in our calculation. On the other hand, Ref [15] uses the Ball-Chiu Ansatz for the $q \bar{q} \gamma$-vertex. Since our results compare favorably with the experimental charge radii, we expect our results for the vector radii to be more realistic than those of Ref. [15].

The quark model [QM] calculations of Refs. [13] and [17] report a considerably smaller value for $\left\langle r_{\rho}^{2}\right\rangle$. Again, this is in part due to the fact that neither of these calculations incorporate the effects of vector meson poles in the quark-photon vertex $[\underline{6}]$.

Another significant difference between our results and those of Ref. [15] is that the latter predicts a zero crossing of $G_{\mathrm{E}}\left(Q^{2}\right)$ at about $Q^{2} \approx 1.7 \mathrm{GeV}^{2}$ for the $\rho$-meson, whereas we do not find any evidence for such a zero crossing below $Q^{2}=2.5 \mathrm{GeV}^{2}$. Extrapolating our numerical results for $G_{\mathrm{E}}\left(Q^{2}\right)$ using a (2,3)-Padé fit suggests a zero crossing around $Q^{2} \approx 3.8 \mathrm{GeV}^{2}$. For comparison, Ref. 13] predicts a zero crossing at $Q^{2} \approx 2.9 \mathrm{GeV}^{2}$, and Ref. 17] at $Q^{2} \approx 5.5 \mathrm{GeV}^{2}$, whereas neither of the sum rule analyses [18] and Ref. [19] predict a zero crossing of $G_{\mathrm{E}}\left(Q^{2}\right)$ below $Q^{2}=5 \mathrm{GeV}^{2}$. Based on our calculations, and on the rather wide range of predictions from other
TABLE III: Our results for the $\rho$ meson electric, magnetic, and quadrupole form factors $G_{\mathrm{E}, \mathrm{M}, \mathrm{Q}}\left(Q^{2}\right)$ at $Q^{2}=1 \mathrm{GeV}^{2}$ and at $Q^{2}=2 \mathrm{GeV}^{2}$, compared to previous DSE [15], lightcone [17], and sum rule calculations [19].

\begin{tabular}{l|ccc|ccc} 
& \multicolumn{3}{|c|}{$Q^{2}=1 \mathrm{GeV}^{2}$} & \multicolumn{3}{c}{$Q^{2}=2 \mathrm{GeV}^{2}$} \\
\hline & $G_{\mathrm{E}}$ & $G_{\mathrm{M}}$ & $G_{\mathrm{Q}}$ & $G_{\mathrm{E}}$ & $G_{\mathrm{M}}$ & $G_{\mathrm{Q}}$ \\
\hline current & 0.22 & 0.57 & -0.11 & 0.08 & 0.27 & -0.05 \\
Ref. [15] & 0.17 & 0.85 & -0.51 & -0.04 & 0.45 & -0.32 \\
Ref. [17] & 0.38 & 0.93 & -0.23 & 0.18 & 0.59 & -0.15 \\
Ref. [19] & 0.25 & 0.58 & -0.49 & 0.13 & 0.28 & -0.24
\end{tabular}

calculations, we conclude that it is unlikely for $G_{\mathrm{E}}\left(Q^{2}\right)$ to have a zero crossing below $Q^{2} \approx 3 \mathrm{GeV}^{2}$.

The values we obtain for the magnetic and quadrupole moments of the $\rho$-meson, $\mu$ and $\mathcal{Q}$, are very similar to those of Ref. [17], and significantly smaller than those of [13] and Refs. 15]. However, the $Q^{2}$ evolution of both $G_{\mathrm{M}}$ and $G_{\mathrm{Q}}$ is quite different than that of Ref. 17] (see Table III), again most likely due to the fact that VMD effects are not properly accounted for in Ref. [17].

A recent sum rule analysis [16] obtained $\mu=2.0 \pm 0.3$ for the magnetic moment, which, given the large error bars, is not accurate enough to discriminate between different calculations. The sum rule analysis of $\rho$ form factors at $Q^{2}=1 \mathrm{GeV}^{2}$ and $2 \mathrm{GeV}^{2}[19]$ seems to support our calculation, see Table III at least for $G_{\mathrm{E}}$ and $G_{\mathrm{M}}$. Our results for the quadrupole form factor however are almost a factor of five smaller than those of Ref. [19]. Clearly the quadrupole form factor is most sensitive to the details of the dynamics.

As we can see in Figs. 5 and 6, not only $G_{\mathrm{E}}\left(Q^{2}\right)$, but also $G_{\mathrm{M}}\left(Q^{2}\right)$ and $G_{\mathrm{Q}}\left(Q^{2}\right)$ have poles in the timelike region as one approaches $Q^{2}=-M_{\rho}^{2}$; in addition, the $K^{\star}$ form factors also have poles at $Q^{2}=-M_{\phi}^{2}$. In Fig. 5 we also show the electromagnetic form factors of a fictitious $s \bar{s}$-like vector meson with the photon coupled (with charge one) to only the $s$-quark, but not to the $\bar{s}$-quark ${ }^{3}$. The form factors of this fictitious $s \bar{s}$-like vector meson have poles at $Q^{2}=-M_{\phi}^{2}$. For comparison we also show a pure VMD model for the electric form factor

$$
G_{\mathrm{E}}\left(Q^{2}\right)=\frac{M_{\mathrm{V}}^{2}}{M_{\mathrm{V}}^{2}+Q^{2}}
$$

for both the $\rho$ and the $s \bar{s}$-like meson in the top panel of Fig. 5. Such a VMD model works remarkably well for the pion electromagnetic form factor, at least up to spacelike $Q^{2}$ values of about $4 \mathrm{GeV}^{2}$. Fig. 5 shows that our form factors of the vector mesons deviate significantly from a simple VMD curve. Only in the timelike region, near the actual vector meson pole, does the VMD curve

\footnotetext{
3 The form factors of the physical $\phi$ meson are trivially zero, as are the form factors of the neutral $\rho$ meson.
} 

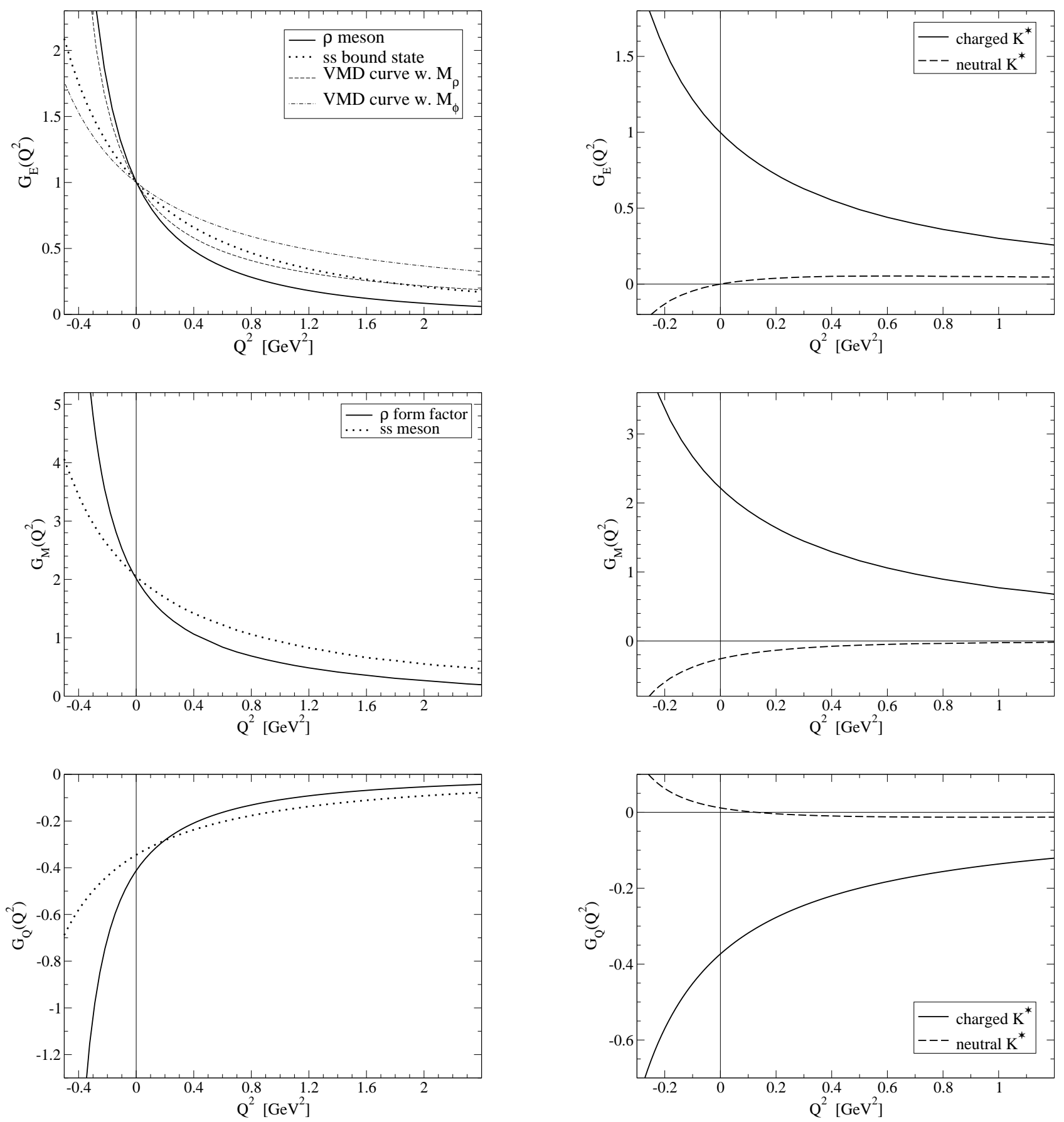

FIG. 5: Numerical results for $G_{\mathrm{E}}\left(Q^{2}\right)$ (top), $G_{\mathrm{M}}\left(Q^{2}\right)$ (middle), and $G_{\mathrm{Q}}\left(Q^{2}\right)$ (bottom) for the $\rho$ meson and a (fictitious) $s \bar{s}$ state. For comparison, we also show the VMD result (dashed and dot-dashed curves) for $G_{\mathrm{E}}\left(Q^{2}\right)$.

resemble our results. In the spacelike region, our results drop significantly faster than a VMD form factor.

Our results for the charged $K^{\star}$ meson form factors are qualitatively similar to those for the $\rho$ meson. The neutral $K^{\star}$ form factors are most sensitive to details of the calculation, since it depends on the difference between

FIG. 6: Numerical results for the neutral and charged $K^{\star}$ form factors $G_{\mathrm{E}}\left(Q^{2}\right)$ (top), $G_{\mathrm{M}}\left(Q^{2}\right)$ (middle), and $G_{\mathrm{Q}}\left(Q^{2}\right)$ (bottom).

the up/down quarks and the strange quarks. Therefore we expect these form factors to show more model dependence than the presented form factors of the charged $K^{\star}$ and $\rho$ mesons, and this is indeed what we see in Table again in particular for the quadrupole moment.

Finally, from Table II it is interesting to note that all studies, with the exception of the light-cone QM calcu- 
lation of Ref. [17], find the charge radii of (charged) vector mesons to be larger than those of the corresponding pseudoscalar mesons. This trend was recently confirmed in lattice calculations for light quarks 21] and for charmonium-like states [20], with the photon coupled to the quark only, not to the antiquark, of the charmonium state. Also a recent non-relativistic QM calculation of such charmonium-like states gives a vector charge radius that is larger than the pseudoscalar charge radius [56]. This means that the vector states are broader than the corresponding pseudoscalar states, assuming that the charge distribution is indicative of the physical size of the bound state. This agrees with the naive intuition that a more tightly bound state is more compact than a heavier state with the same constituents.

\section{B. Quark-mass dependence}

In addition to the electromagnetic form factors of the physical $\rho$ meson, we have also calculated the quarkmass dependence of these form factors. For simplicity we restrict ourselves to equal-mass mesons, i.e. $q \bar{q}$ bound states, and for the electromagnetic properties we couple the photon to only the quark $q$ (with charge one), not to the antiquark $\bar{q}$. We refer to these form factors as the single-quark form factors; even though they are unphysical, they are well-defined and allow for comparisons with other theoretical and computational studies of the vector meson form factors.

The static electromagnetic properties are plotted in Fig. 7 as function of the current quark mass, normalized by the up/down current quark mass; for the strange quark we have $m_{s} \approx 23 m_{u / d}$ and for the charm mass $m_{c} \approx 224 m_{u / d}$. Our numerical errors grow with increasing meson mass (i.e. increasing quark mass), mainly because the momentum $p^{2}$ of the quark propagator in the Bethe-Salpeter integrals and in the triangle diagram spans an increasingly large domain in the complex $p^{2}$ plane with increasing meson mass. The analytic continuation of the solution of the quark DSE, Eq. (1), from the spacelike (Euclidean) axis to this complex momentum domain becomes numerically cumbersome and inaccurate for large meson masses. In our calculations this is reflected by the fact that for the charm quark we have an estimated $2 \%$ to $4 \%$ numerical uncertainty in the moments, and a numerical uncertainty of $6 \%$ to $8 \%$ in the radii.

The magnetic moment $\mu$ turns out to be almost independent of the current quark mass, Fig. 7 For $0<$ $m_{q}<4 m_{s} \approx 100 m_{u / d}$ the magnetic moment increases with quark mass from $\mu=2.01$ to $\mu=2.12$; above $4 m_{s}$ the numerical uncertainty starts to increase and the magnetic moment is basically independent of the quark mass within our numerical error bars of a few percent.

This mass dependence can naively be understood by considering a simple constituent quark model, in which a vector meson is a bound state of two quarks in an S-wave
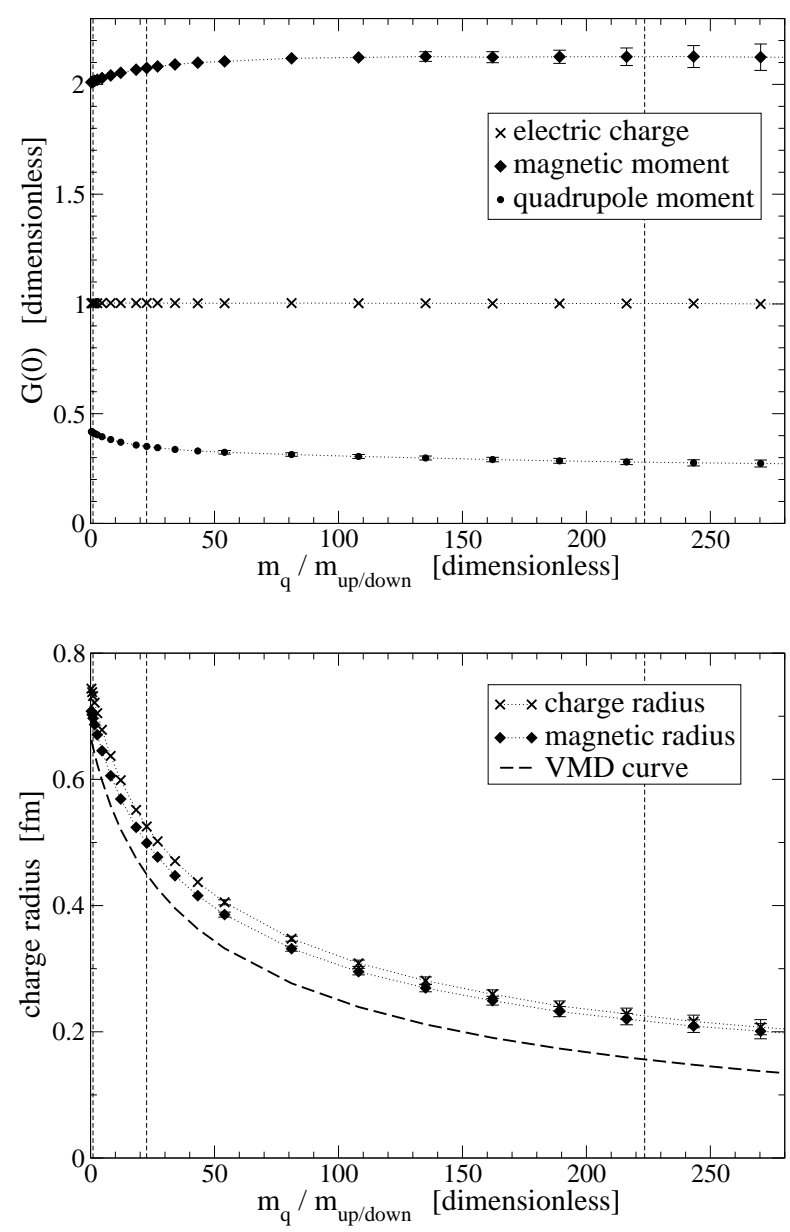

FIG. 7: Numerical results for the magnetic and quadrupole moments (dimensionless, top) and for the charge radius (in fermi, bottom) as function of the current quark mass. Vertical dashed lines indicate the up/down, strange, and charm quark masses.

with the spins aligned. In such a model one expects the magnetic moment to be proportional to that of its constituents, i.e. $\mu_{\mathrm{V}} \propto \mu_{q}$, where $\mu_{q}$ is the magnetic moment of the constituent quark. As long as the quark magnetic moment is only weakly dependent on the current quark mass, so is the magnetic moment of the bound state.

Since both the charge, $G_{\mathrm{E}}(0)$, and the magnetic moment, $G_{\mathrm{M}}(0)$, are (almost) independent of the quark mass, one might expect that also the quadrupole moment, $G_{\mathrm{Q}}(0)$, depends only weakly on the quark mass. However, our calculation shows that this is not the case: The quadrupole moment $\mathcal{Q}$ decreases monotonically with $m_{q}$, and is reduced by about $25 \%$ at $m_{q} \approx 2 m_{s} \approx$ $50 m_{u / d}$. Above this quark mass, the quadrupole moment continues to decrease with quark mass, but at a slower rate. The mass dependence of the quadrupole moment may not be that surprising on realizing that our results, $-0.41<\mathcal{Q}<-0.27$ depending on quark mass, deviate significantly from the canonical value, $\mathcal{Q}=-1$. 
Furthermore, of all the static electromagnetic properties, the quadrupole moment shows the largest model dependence, see Table II indicating that the details of the dynamics are important for this quantity.

The fact that we find a nontrivial quadrupole moment for all quark masses, indicates that the naive picture of a vector meson as a nonrelativistic bound state of two quarks in an S-wave is too simple. Clearly, there is a significant amount of quark orbital momentum in the vector mesons, which in our approach is incorporated in the meson BSAs. This is related to the fact that we use method that is explicitly frame-independent: orbital angular momentum is not a Poincaré invariant quantity.

In the bottom panel of Fig. 7 we show the mass dependence of the charge and magnetic radii (defined analogously) of the vector mesons. Just like the pion charge radius [49], both the charge and the magnetic radius decreases with increasing quark mass. Our results for $\left\langle r^{2}\right\rangle^{\frac{1}{2}}$ are qualitatively similar to a VMD curve, and over the entire mass range the charge radius can in fact be reasonably well described by a VMD curve with a constant shift of about $0.07 \mathrm{fm}$. Of course that means that the relative deviation from a VMD model increases with increasing quark mass, again just as in the case of the pion form factor.

Finally, we consider the single-quark transition form factors of the $\eta_{c}$ and $J / \Psi$ mesons, at $m_{c} \approx 224 m_{u / d}$. This allows us to compare our results with the lattice simulations of Ref. [20]. Both the magnetic moment and the quadrupole moment are in good agreement with the lattice data, see the bottom row of Table [I] Also the charge radii are in reasonable agreement, with the vector state being slightly larger than the pseudoscalar state.

In this paper we are interested in the quark-core contribution to mesonic observables, and do not include pion loop effects. However, the form factors, and their quarkmass dependence, are sensitive to pion loops, in particular at small quark masses. Pion loops lead to corrections to the physical $\rho$ and $\pi$ charge radii of about $10 \%$ to $15 \%$ [57, 58], but their contributions decrease rapidly with increasing quark mass. Similarly, we expect that at the physical up/down quark masses, the other form factors, $G_{\mathrm{M}}$ and $G_{\mathrm{Q}}$ will also receive corrections of the order of $10 \%$ due to pion loops. That means that the mass dependence of the magnetic moment will be dominated by the pion loop corrections, given the very weak quark-mass dependence we find here. However, we expect these corrections to become negligible for masses around $m_{s}$ and above.

\section{CONCLUSIONS}

We have calculated the electromagnetic form factors of the $\rho$ meson, and of both the charged and the neutral $K^{\star}$ mesons. Our method is explicitly Poincaré invariant, and we have demonstrated explicitly that physical observables are frame-independent. By dressing the quark- photon vertex we guarantee electromagnetic current conservations; furthermore, our dressed quark-photon vertex exhibits poles in the timelike region, corresponding to intermediate vector mesons. Exactly the same method, using the same model for the effective interaction 22], has been used quite successfully to describe the pion electromagnetic form factor [7, 22] and a plethora of other light meson observables within about $10 \%$ to $15 \%$ [27]. We therefore expect our results to have a similar level of accuracy.

Compared to other calculations, we find a stronger $Q^{2}$ dependence, mostly due to the fact that we have incorporated unambiguously VMD effects in the quark-photon vertex, which have been neglected in Refs. [13, 15, 17]. Our results favor a magnetic moment close to two, in agreement with sum rule analysis [16, 19], and a quadrupole moment $\mathcal{Q} \approx-0.4$, which is similar to the result of Ref. [17], but significantly smaller than suggested by other calculations [13, 15, 19]. Our results for the quadrupole moment indicate that there is a significant amount of quark orbital angular momentum in the vector mesons.

The magnetic moment is almost independent of the quark mass for mesons of equal-mass constituents. The quadrupole moment decreases with increasing quark mass. Also the shape of the form factors changes: since the VMD pole in the timelike region shifts further away from $Q^{2}=0$ with increasing quark mass, the form factors become less steep, and the radii decrease with increasing quark mass. Over the entire quark mass range, from the chiral limit to the charm quark mass, the charge radius can be reasonably described by a VMD curve with a constant shift of about $0.07 \mathrm{fm}$. A similar behavior of the form factors, and of their quark-mass dependence, has been obtained using the model of Ref. [59] for the effective quark-antiquark interaction.

Ideally experiments would guide us in order to discriminate between different models and different calculation methods, but it is unlikely that these form factors can be measured in the near future. Lacking reliable experimental input, it would be very useful to have (quenched) lattice data for the vector meson form factors at light quark masses. Currently, the only accurate lattice data available are at the charm mass [20]; these lattice data are in reasonable agreement with our results at $m_{c}$. Accurate lattice simulations at light quark masses are needed in order to discriminate between different model calculations. A beginning has been made in Ref. [21], but the error bars have to be reduced significantly in order to make a detailed comparison meaningful.

\section{ACKNOWLEDGMENTS}

We would like to thank Jo Dudek, Eric Swanson, Peter Tandy, and in particular Craig Roberts for useful discussions and encouragement. This work was supported by the US Department of Energy, contract No. DE-FG02- 
00ER41135, and the Department of Energy, Office of Nuclear Physics, contract No. DE-AC02-06CH11357. The work benefited from the facilities of the NSF Terascale Computing System at the Pittsburgh Supercomputing Center.

\section{APPENDIX A: NUMERICAL APPROACH}

The form factors are calculated in impulse approximation

$$
\begin{aligned}
\Lambda_{\mu, \rho \sigma}^{a a \bar{b}}(P, Q)= & i N_{c} \int_{k} \operatorname{Tr}\left[\Gamma_{\mu}^{a}\left(k_{-}, k_{+}\right) \chi_{\rho}^{a \bar{b}}\left(k_{+}, k_{P}\right)\right. \\
& \left.\times S^{b}\left(k_{P}\right)^{-1} \bar{\chi}_{\sigma}^{\bar{b} a}\left(q, k_{-}\right)\right]
\end{aligned}
$$

with $k_{P}=k-P / 2$ and $k_{ \pm}=k+P / 2 \pm Q / 2$. That means that we need numerical solutions of the rainbowladder BSE for

- quark-photon vertex

- incoming vector meson

- outgoing vector meson

in addition to the solution of the quark DSE in rainbow truncation. If we choose the momentum routing and integration variables carefully, we can arrange the integration grids such that we do not need any interpolation or extrapolation in the the final (triangle) loop integral for the form factor. That significantly reduces numerical errors, in particular possible systematic errors introduced by the extrapolation.

We use the momentum frame

$$
\begin{aligned}
P_{\mu} & =(0,0,0, P) \\
Q_{\mu} & =(Q, 0,0,0)
\end{aligned}
$$

with $P^{2}+\frac{1}{4} Q^{2}=-M^{2}$. Depending on the value of $Q^{2}$, either $P$ or $Q$ or both are imaginary. In addition, we have the integration momentum

$$
k_{\mu}=k(\cos (\theta), \sin (\theta) \cos (\phi), \sin (\theta) \sin (\phi), 0),
$$

and corresponding measure

$$
\int \frac{d^{4} k}{(2 \pi)^{4}}=\int_{0}^{\infty} \frac{k^{3} d k}{(2 \pi)^{3}} \int_{0}^{\pi} \sin ^{2}(\theta) d \theta \int_{0}^{\pi} \sin (\phi) d \phi .
$$

We use Eqs. (16)-(18) for the general structure of the coupling of a photon to an on-shell vector meson, and perform the traces analytically to obtain expressions for the form factors $F_{i}\left(Q^{2}\right)$. Subsequently, we use Eqs. (23)(25) to convert the functions $F_{i}$ to the more conventional electric, magnetic, and quadrupole form factors $G_{\mathrm{E}}, G_{\mathrm{M}}$, and $G_{\mathrm{Q}}$.

\section{Quark-photon vertex}

The $q \bar{q} \gamma$-vertex is the solution of an inhomogeneous BSE, which in ladder truncation we can write as

$$
\begin{aligned}
\Gamma_{\mu}\left(k_{-}, k_{+} ; Q\right)= & Z_{2} \gamma_{\mu}+\frac{4}{3} \int \frac{d^{4} q}{(2 \pi)^{4}} 4 \pi \alpha\left((k-q)^{2}\right) \\
& \times D_{\rho \sigma}(k-q) \gamma_{\rho} \chi_{\mu}\left(q_{-}, q_{+} ; Q\right) \gamma_{\sigma}
\end{aligned}
$$

where

$$
\chi_{\mu}\left(q_{-}, q_{+} ; Q\right)=S\left(q_{-}\right) \Gamma_{\mu}\left(q_{-}, q_{+} ; Q\right) S\left(q_{+}\right)
$$

with $k_{ \pm}=k+P / 2 \pm Q / 2$, and similarly for $q_{ \pm}$. Both $k$ and $q$ are real Euclidean vectors, and the incoming and outgoing quarks are always of the same flavor, so we can drop any flavor indices.

In order to solve such a BSE, we decompose the function $\chi$ into its Dirac components, and project out a set of coupled integral equations for its scalar component functions $F_{i}$. (Equivalently, one could decompose $\Gamma$ into its Dirac components, and solve the coupled integral equations for its components, but it turns out that solving the BSE for $\chi$ is is roughly factor of three faster than solving the BSE for $\Gamma$.) We solve these coupled integral equations by iteration, after discretizing the angular and radial variables.

The most general form of the quark-photon vertex requires twelve Dirac structures. Four of these covariants represent the longitudinal components which are completely specified by the Ward-Takahashi identity in terms of the (inverse) quark propagator and they do not contribute to elastic form factors. The transverse part of the vertex $\chi$ can be decomposed into eight components

$$
\begin{aligned}
& \chi_{\mu}\left(k_{-}, k_{+} ; Q\right)= \\
& \quad \sum_{i=1}^{8} T_{i}\left(k+\frac{1}{2} P, Q\right) F_{i}\left(k^{2}, \theta, \phi\right),
\end{aligned}
$$

where the functions $F_{i}$ depend on two angles, because we solve it in exactly the same frame and variables as it is used in the loop integral for the form factor; alternatively, we could write it as a function of $k^{2}, k \cdot P$, and $k \cdot Q$.

\section{Vector meson BSAs}

The incoming vector meson, with momentum $P-\frac{1}{2} Q$ and flavor labels $a \bar{b}$, is the solution of the homogeneous BSE

$$
\begin{aligned}
& S_{a}^{-1}\left(k_{+}\right) \chi_{\rho}^{a \bar{b}}\left(k_{+}, k_{P} ; P-\frac{1}{2} Q\right) S_{b}^{-1}\left(k_{P}\right)= \\
& \quad \frac{4}{3} \int \frac{d^{4} q}{(2 \pi)^{4}} 4 \pi \alpha\left((k-q)^{2}\right) D_{\alpha \beta}(k-q) \\
& \quad \times \gamma_{\alpha} \chi_{\rho}^{a \bar{b}}\left(q_{+}, q_{P} ; P-\frac{1}{2} Q\right) \gamma_{\beta},
\end{aligned}
$$


with $k_{+}=k+P / 2+Q / 2, k_{P}=k-P / 2$, and similarly for $q_{+}$and $q_{P}$. The vector meson is on its mass-shell: $P^{2}+\frac{1}{4} Q^{2}=-M^{2}$.

Again, we decompose the transverse vertex function $\chi$ into eight components, but use a slightly different decomposition than for the quark-photon vertex, because the momentum arguments are different. For the vector meson BSAs we use

$$
\begin{aligned}
& \chi_{\rho}\left(k_{+}, k_{P} ; P-\frac{1}{2} Q\right)= \\
& \quad \sum_{i=1}^{8} T_{i}\left(k_{P}, P-\frac{1}{2} Q\right) F_{i}\left(k^{2}, \theta, \phi\right),
\end{aligned}
$$

i.e. we use the momentum partitioning where the total incoming meson momentum flows into the outgoing quark leg; or in other words, we use the incoming quark momentum as the "relative momentum" in the decomposition $(\eta=1)$, though not as the integration momentum. And again, the functions $F_{i}$ depend on two angles. As a check on our numerics, we calculate the leptonic decay constant in this frame as well, which gives us an indication of the numerical errors in the BSAs.
We do not explicitly use any algebraic relation between $\chi$ and $\bar{\chi}$

$$
\bar{\chi}(p, P)=\left[C^{-1} \chi(-p,-P) C\right]^{\text {transpose }},
$$

because the arguments of $\chi$ and $\bar{\chi}$ are different. Instead, we simply solve both for $\chi$ and for $\bar{\chi}$ in the appropriate frame. That is, we calculate the BSA of the outgoing vector meson as the solution of

$$
\begin{aligned}
& S_{b}^{-1}\left(k_{P}\right) \bar{\chi}_{\sigma}^{\bar{b} a}\left(k_{P}, k_{-} ; P+\frac{1}{2} Q\right) S_{a}^{-1}\left(k_{-}\right)= \\
& \quad \frac{4}{3} \int \frac{d^{4} q}{(2 \pi)^{4}} 4 \pi \alpha\left((k-q)^{2}\right) D_{\alpha \beta}(k-q) \\
& \quad \times \gamma_{\alpha} \bar{\chi}_{\beta}^{\bar{b} a}\left(q_{P}, q_{-} ; P+\frac{1}{2} Q\right) \gamma_{\beta},
\end{aligned}
$$

again with $k_{-}=k+P / 2-Q / 2, k_{P}=k-P / 2$, and similarly for $q_{-}$and $q_{P}$. We solve this equation for $\bar{\chi}$ in basically the same manner as the BSE for $\chi$. This way we avoid the need for interpolation and extrapolation on the vertex functions in the triangle diagram for the electromagnetic form factors.
[1] G.R. Farrar and D.R. Jackson, Phys. Rev. Lett. 43, 246 (1979).

[2] V.A. Nesterenko and A.V. Radyushkin, Phys. Lett. B 115, 410 (1982).

[3] C.D. Roberts, Nucl. Phys. A 605, 475 (1996) arXiv:hep-ph/9408233.

[4] C.J. Burden, C.D. Roberts and M.J. Thomson, Phys. Lett. B 371, 163 (1996) arXiv:nucl-th/9511012.

[5] H.M. Choi and C.R. Ji, Phys. Rev. D 59, 074015 (1999) arXiv:hep-ph/9711450.

[6] P. Maris and P.C. Tandy, Phys. Rev. C 61, 045202 (2000) arXiv:nucl-th/9910033.

[7] P. Maris and P.C. Tandy, Phys. Rev. C 62, 055204 (2000) arXiv:nucl-th/0005015.

[8] C.W. Hwang, Phys. Rev. D 64, 034011 (2001) arXiv:hep-ph/0105016.

[9] A.P. Bakulev, K. Passek-Kumericki, W. Schroers and N.G. Stefanis, Phys. Rev. D 70, 033014 (2004) [Erratumibid. D 70, 079906 (2004)] arXiv:hep-ph/0405062.

[10] J. Volmer et al. [The Jefferson Lab F(pi) Collaboration], Phys. Rev. Lett. 86, 1713 (2001) arXiv:nucl-ex/0010009.

[11] V. Tadevosyan et al. [Fpi-1 Collaboration], "Determination of the pion charge form factor for $\mathrm{Q}^{* *} 2=0.60$ $1.60(\mathrm{GeV} / \mathrm{c})^{* *} 2, "$ arXiv:nucl-ex/0607007; T. Horn et al. [Fpi2 Collaboration], "Determination of the charged pion form factor at Q2 $=1.60$ and $2.45(\mathrm{GeV} / \mathrm{c}) 2$," arXiv:nucl-ex/0607005.

[12] F. Cardarelli, I. L. Grach, I. M. Narodetsky, G. Salme and S. Simula, Phys. Lett. B 349, 393 (1995) arXiv:hep-ph/9502360.

[13] J.P.B. de Melo and T. Frederico, Phys. Rev. C 55, 2043 (1997) arXiv:nucl-th/9706032.

[14] M. B. Hecht and B. H. J. McKellar, Phys. Rev. C 57, 2638 (1998) arXiv:hep-ph/9704326.
[15] F.T. Hawes and M.A. Pichowsky, Phys. Rev. C 59, 1743 (1999) arXiv:nucl-th/9806025.

[16] A. Samsonov, JHEP 0312, $061 \quad$ (2003) arXiv:hep-ph/0308065.

[17] H.M. Choi and C.R. Ji, Phys. Rev. D 70, 053015 (2004) arXiv:hep-ph/0402114.

[18] V.V. Braguta and A.I. Onishchenko, Phys. Rev. D 70, 033001 (2004) arXiv:hep-ph/0403258.

[19] T.M. Aliev and M. Savci, Phys. Rev. D 70, 094007 (2004) arXiv:hep-ph/0405235.

[20] J.J. Dudek, R.G. Edwards and D.G. Richards, Phys. Rev. D 73, 074507 (2006) arXiv:hep-ph/0601137.

[21] B.G. Lasscock, J. Hedditch, D.B. Leinweber and A.G. Williams, arXiv:hep-lat/0611029.

[22] P. Maris and P.C. Tandy, Phys. Rev. C 60, 055214 (1999) arXiv:nucl-th/9905056.

[23] D. Jarecke, P. Maris and P.C. Tandy, Phys. Rev. C 67, 035202 (2003) arXiv:nucl-th/0208019.

[24] P. Maris and P.C. Tandy, Phys. Rev. C 65, 045211 (2002) arXiv:nucl-th/0201017.

[25] C.D. Roberts and S.M. Schmidt, Prog. Part. Nucl. Phys. 45S1, 1 (2000) arXiv:nucl-th/0005064.

[26] R. Alkofer and L. von Smekal, Phys. Rept. 353, 281 (2001) arXiv:hep-ph/0007355.

[27] P. Maris and C.D. Roberts, Int. J. Mod. Phys. E 12, 297 (2003) arXiv:nucl-th/0301049.

[28] C.S. Fischer, J. Phys. G 32, R253 (2006) arXiv:hep-ph/0605173.

[29] A. Bender, C.D. Roberts and L. Von Smekal, Phys. Lett. B 380, 7 (1996) arXiv:nucl-th/9602012; A. Bender, W. Detmold, C.D. Roberts and A.W. Thomas, Phys. Rev. C 65, 065203 (2002) arXiv:nucl-th/0202082.

[30] P. Maris, C.D. Roberts and P.C. Tandy, Phys. Lett. B 420, 267 (1998) arXiv:nucl-th/9707003.

[31] P. Maris and C.D. Roberts, Phys. Rev. C 56, 3369 (1997) 
arXiv:nucl-th/9708029.

[32] P. Maris, arXiv:nucl-th/0611057

[33]

[34] W.M. Yao et al. [Particle Data Group], J. Phys. G 33, 1 (2006).

[35] K.W. Edwards et al. [CLEO Collaboration], Phys. Rev. Lett. 86, 30 (2001) arXiv:hep-ex/0007012.

[36] reference to insensitivity of results to variations in parameters by CDR et al.

[37] A. Holl, A. Krassnigg, P. Maris, C.D. Roberts and S.V. Wright, Phys. Rev. C 71, 065204 (2005) arXiv:nucl-th/0503043.

[38] J. Skullerud, D.B. Leinweber and A.G. Williams, Phys. Rev. D 64, 074508 (2001) arXiv:hep-lat/0102013;

[39] P.O. Bowman, U.M. Heller, D.B. Leinweber and A.G. Williams, Nucl. Phys. Proc. Suppl. 119, 323 (2003) arXiv:hep-lat/0209129.

[40] P.O. Bowman, U.M. Heller, D.B. Leinweber, A.G. Williams and J.B. Zhang, Nucl. Phys. Proc. Suppl. 128 (2004) 23 arXiv:hep-lat/0403002.

[41] M.S. Bhagwat, M.A. Pichowsky, C.D. Roberts and P.C. Tandy, Phys. Rev. C 68, 015203 (2003) arXiv:nucl-th/0304003.

[42] M.S. Bhagwat, A. Holl, A. Krassnigg, C.D. Roberts and P.C. Tandy, Phys. Rev. C 70, 035205 (2004) arXiv:nucl-th/0403012.

[43] H.H. Matevosyan, A.W. Thomas and P.C. Tandy, arXiv:nucl-th/0605057

[44] J. Skullerud and A. Kizilersu, JHEP 0209, 013 (2002) arXiv:hep-ph/0205318.

[45] J.I. Skullerud, P.O. Bowman, A. Kizilersu, D.B. Lein- weber and A.G. Williams, JHEP 0304, 047 (2003) arXiv:hep-ph/0303176.

[46] J.I. Skullerud, P.O. Bowman, A. Kizilersu, D.B. Leinweber and A.G. Williams, Nucl. Phys. Proc. Suppl. 141, 244 (2005) arXiv:hep-lat/0408032.

[47] M.S. Bhagwat and P.C. Tandy, Phys. Rev. D 70, 094039 (2004) arXiv:hep-ph/0407163.

[48] F.J. Llanes-Estrada, C.S. Fischer and R. Alkofer, Nucl. Phys. Proc. Suppl. 152, 43 (2006) arXiv:hep-ph/0407332.

[49] P. Maris and P.C. Tandy, arXiv:nucl-th/0511017

[50] D.R. Yennie, Phys. Rev. Lett. 34, 239 (1975).

[51] M.N. Achasov et al., Phys. Rev. Lett. 86, 1698 (2001) arXiv:hep-ex/0102029.

[52] M.S. Bhagwat, A. Krassnigg, P. Maris and C.D. Roberts, arXiv:nucl-th/0612027

[53] R.G. Arnold, C.E. Carlson and F. Gross, Phys. Rev. C 21, 1426 (1980).

[54] S.J. Brodsky and J.R. Hiller, Phys. Rev. D 46, 2141 (1992).

[55] J.S. Ball and T. W. Chiu, Phys. Rev. D 22, 2542 (1980).

[56] O. Lakhina and E.S. Swanson, Phys. Rev. D 74, 014012 (2006) arXiv:hep-ph/0603164.

[57] R. Alkofer, A. Bender and C.D. Roberts, Int. J. Mod. Phys. A 10, 3319 (1995) arXiv:hep-ph/9312243.

[58] M.A. Pichowsky, S. Walawalkar and S. Capstick, Phys. Rev. D 60, 054030 (1999) arXiv:nucl-th/9904079.

[59] R. Alkofer, P. Watson and H. Weigel, Phys. Rev. D 65, 094026 (2002) arXiv:hep-ph/0202053. 